\title{
The effect of three-dimensional imaging of well-known objects on time and accuracy of mental rotation
}

\begin{abstract}
BACKGROUND
The purpose of this study was to verify hypotheses concerning the effect of three-dimensional imaging and the canonicity of objects presented in the original position on the reaction time (RT) and the accuracy $(\mathrm{A})$ of mental rotation task (MRT) execution. The classical paradigm of MRT, developed by Shepard and Metzler (1971), was used in the experiment.
\end{abstract}

\section{PARTICIPANTS AND PROCEDURE}

One hundred fifty-eight undergraduate students ( $88 \mathrm{fe}$ male and 70 male), aged 18-30 years, participated in the experiment. All participants had normal vision or corrected vision, and reported no stereo blindness. The sequential version of the MRT was used in the experiment. Participants answered whether the object observed in the second position was only rotated or both rotated and mirror-reversed, in comparison to its original position. The answer (accuracy) and its latency (RT) were recorded.

\section{RESULTS}

As predicted by the mental rotation model, both the "U"shaped A-MRT distribution and the inverted " $U$ "-shaped
RT-MRT distribution were found, due to the angular disparity. For the RT-MRT, this effect was more pronounced when the objects were displayed stereoscopically than in a plane, and when the objects were presented in the original position from the canonical orientation rather than an unusual point of view. On the other hand, in the case of the A-MRT, an effect of the orientation of objects presented in the original position on strengthening the relationship between accuracy and angular disparity was found.

\section{CONCLUSIONS}

The results indicated that the interactions between the presentation of the objects in the mental rotation task (stereoscopically vs. in a plane) and the orientation of the object in its original position (canonically vs. unusual) are more complicated than would appear from predictions of classical theories of mental rotation. The results of this study are discussed in relation to the theories of recognition and categorization.

\section{KEY WORDS}

mental rotation; canonical orientation; stereoscopic presentation; three-dimensional natural objects

organizations - Faculty of Social Sciences, Department of Experimental Psychology, John Paul II Catholic University of Lublin, Poland

AUthors' CONTRIBUtion - A: Study design - B: Data collection - C: Statistical analysis - D: Data interpretation . E: Manuscript preparation · F: Literature search · G: Funds collection

CORRESPONDING AUthor - Prof. Piotr Francuz, Faculty of Social Sciences, Department of Experimental Psychology, John Paul II Catholic University of Lublin, 14 Racławickie Avenue, 20-950 Lublin, Poland, tel. +48 8144535 09, e-mail: francuz@kul.pl

TO CITE THIS ARTICLE - Francuz, P. (2014). The effect of three-dimensional imaging of well-known objects on time and accuracy of mental rotation. Health Psychology Report, 2(2), 90-98. DOI: 10.5114/hpr.2014.43915 


\section{BACKGROUND}

It is assumed that the answer to the question whether two objects presented from different points of view are the same or different (e.g. mirror-reflected) is a proper operationalization of an imaginary process of mental rotation (Shepard \& Metzler, 1971). This task, known as "Shepard-Metzler's Mental Rotation Task" (MRT), has two variants: sequential and simultaneous. In a sequential task, the first (target) object of the pair is presented in the original position and a second one is presented in a rotated or rotated and mirror-reflected position. According to this procedure, both objects are presented separately, one after the other. This means that when the second object of a pair is presented, the participant of an experiment must compare its shape with a stored image of the first object. In the simultaneous task, both items are presented together, side by side. The reaction time (RT) and accuracy (A) of the decision whether the two items match are correlated with the degree of angular disparity between the target and rotated object. These two parameters (RT-MRT and A-MRT) are interpreted as indicators of the imaginative process of rotation, analogous to the process of rotation of objects in the real world (Shepard \& Cooper, 1982).

Artificial or natural (real-world), known or unknown, two- or three-dimensional stimuli are used in the research of mental rotation. Artificial two- or three-dimensional objects, designed exclusively for use in the experiment, presented in a plane (paper, computer monitor, or projected image on the screen) have been used so far in most studies on mental rotation. In almost all experiments in which participants mentally rotate real-world objects such as chairs, lamps, or humans, these objects are presented on flat, uniform white, gray, or black backgrounds (e.g. Kanamori \& Takeda, 2003; Konkle \& Oliva, 2011; Murray, 1997; Petit, Pegna, Mayer \& Hauert, 2003; Zacks, Mires, Tversky \& Hazeltine, 2002). Much less research has been conducted on the rotation of well-known, real-world objects presented stereoscopically (Konkle \& Oliva, 2011; Lawson \& Jolicoeur, 2003; Noudoost, Adibi, Moeeny \& Esteky, 2005; Ruddle \& Jones, 2001; Shepard \& Cooper, 1982; Zacks et al., 2002).

The division of objects into two- or three-dimensional stimuli is based on the arbitrary decisions of the researchers interested in mental rotation processes. For example, Jolicoeur, Regehr, Smith and Smith (1985) and Shepard and Metzler (1988) treated projections of three-dimensional objects onto a plane as three-dimensional objects, while in other studies they have been interpreted as two-dimensional (e.g. McWilliams, Hamilton \& Muncer, 1997; Neubauer, Bergner \& Schatz, 2010; Parsons, 1995; Robert \& Chevrier, 2003). Bauer and Jolicoeur (1996) found that the MRT execution on two-dimensional objects is faster and more accurate than on three-dimensional ones. It was also found that the three-dimensional imaging of objects improves the A-MRT compared to the A-MRT for the same set of objects presented in a plane (e.g., photographs), but does not affect the RT-MRT (Aitsiselmi \& Holliman, 2009; Gaggioli \& Breining, 2001; McWilliams et al., 1997; Neubauer et al., 2010; Parsons, 1995). In turn, Robert and Chevrier (2003) found that the MRT execution on threedimensional imaging objects is both faster and more accurate than when objects are presented in a plane. Referring to these results and because we see such objects in three-dimensional space in our daily experience, it is supposed that the RT-MRT should be shorter and the A-MRT should be higher for objects displayed stereoscopically in comparison to objects displayed in a plane.

The concept of spatial orientation of three-dimensional stimuli requires more detailed discussion. The orientation of an object is defined by the angle at which it is seen at a particular moment. A wellknown (familiar) object can be oriented canonically or non-canonically (unusually), with respect to the observer (Palmer, Rosch \& Chase, 1981). In the discussion on the criteria for canonicity of object orientation, the following issues are highlighted: typicality, time of recognition or categorization and familiarity of the given objects. Firstly, canonically oriented objects are most often encountered in everyday observations. In this sense, we keep in memory the most common views of these objects. Secondly, recognition and categorization of an object presented in canonical orientation is much faster than in any other orientation (Edelman \& Bülthoff, 1992; Jolicoeur, 1985; Palmer et al., 1981; Tarr, 1995). Thirdly, if the object is seen from the canonical or unusual point of view, it can be assumed that the observer has a concept (mental representation) of that object. It is impossible to recognize the canonical orientation of an object without knowledge of it, having no experience of it, having no concept of its shape or functions, or other characteristics that define its identity (Palmer, 1999).

In research on the recognition, categorization, and mental rotation of three-dimensional objects presented from different points of view, there are at least two theories of canonical orientation which overlap to some extent.

On the one hand, in the reports regarding the recognition and categorization processes, it is emphasized that the similarity in orientation between the object and the prototype is an important factor that affects the membership of that object in a category (Edelman, 1995; Palmer et al., 1981). Canonical orientation is usually one of the properties of category prototype. The angular disparity between the canonical orientation of the prototype and orientation of the categorized object similarly affects RT and error
Rotation of well-known objects 
rates of its categorization, such as the differences between an object and prototype in terms of their shape or components (Cutzu \& Edelman, 1994, 1998). This idea is also well known as the rotate-for-recognition hypothesis (Biederman \& Gerhardstein, 1993; Tarr, 1995; Tarr \& Pinker, 1989).

On the other hand, in research on mental rotation of three-dimensional objects, the term "canonical orientation" most often refers to the orientation of the target object presented in the original position ( $0^{\circ}$ rotation). Therefore, every object presented as rotated (the second one from the pair: original-rotated) is seen from the non-canonical (unusual) point of view. It is assumed that a three-dimensional target object is presented in an upright orientation and face-forward, or at least between a frontal and side view (Corballis, 1988; Graf, 2006; Just, Carpenter, Maguire, Diwadkar \& McMains, 2001; Zacks \& Michelon, 2005), and its mental representation is stored in memory also in this orientation (Bülthoff, Edelman \& Tarr, 1995; Just et al., 2001; Palmer et al., 1981; Shepard \& Cooper, 1982; Tarr \& Pinker, 1989).

The purpose of the MRT is primarily to determine whether the two images of the object observed in the two positions (original and rotated) are appearances of the same object. Thus, from this point of view, canonicity alone should not be a factor that affects the time or the accuracy of the MRT execution. However, if it was proven that the canonicity of the object presented in the original position affects the RT-MRT or A-MRT, it should be assumed that the MRT outcome is either (1) a manifestation of a mental process other than a mental rotation (e.g. categorization or recognition), or (2) that the process of mental rotation cannot be separated from categorization and/or recognition processes.

Bülthoff et al. (1995), Edelman and Bülthoff (1992), and Cutzu and Edelman (1998) argue that the representation of the three-dimensional object is stored in one's memory as linked collections of simplified two-dimensional "snapshots" taken from different points of view. According to this idea, angular disparity could be treated as a measure of similarity between a two-dimensional image of a given threedimensional object, presented from some point of view to the two-dimensional image of the prototype of the category. On the one hand, Cutzu and Edelman (1994) argue that the categorization of two-dimensional images representing different points of view of a three-dimensional object is a better model of the mechanism of object recognition than mental rotation. On the other hand, if angular disparity affects RT-MRT and/or A-MRT, this might be because people do not rotate an object in their imagination during the MRT execution, but they classify two-dimensional images of the object after rotation into the category in which a prototype is an object represented by its two-dimensional image in its original position. This would mean that the result of the MRT is a manifestation of categorization mechanisms, rather than spatial imagination. As the MRT is not required to answer the question concerning knowledge of a stimulus (to recognize it), it is not justifiable to say that the RTMRT is a function of its familiarity (Tarr, 1995). The hypothesis of a common neural mechanism for mental rotation and categorization has been repeatedly verified (e.g., Farah, 1990; Turnbull \& McCarthy, 1996; Warrington \& Taylor, 1973). In particular, Shendan and Stern $(2007,2008)$ found that during mental rotation those brain structures are active that are also active during categorization but not during recognition.

The purpose of this study was to verify the hypotheses on the differences in time and accuracy of the MRT execution for the same well-known, real-world three-dimensional objects presented stereoscopically or in a plane. The orientation of objects during their presentation in the original position was manipulated in the present study. Two independent groups of participants took part in the experiment and they executed the same MRT with the same objects. The only difference between groups was the orientation of the objects presented in the original position. In one group the objects were presented from the canonical point of view, and in the other from an unusual point of view. The RT-MRT and the A-MRT were registered in both groups. It was predicted that if the result of the MRT is an indicator of mental rotation, the RTMRT and A-MRT should be similar in the two groups. However, if the differences between groups turned out to be significant, it would support the hypothesis in which the outcome of MRT is an indicator of the process of categorization, recognition, or both, rather than an indicator of the mental rotation.

\section{PARTICIPANTS AND PROCEDURE}

\section{PARTICIPANTS}

Forty-one undergraduate students (22 female and 19 male), aged $20-30$ years $(M=22.63, S D=2.36)$, participated in the preliminary study, which aimed to select experimental stimuli. Participants were recruited from the same population of undergraduate students who participated in the experiment.

One hundred fifty-eight undergraduate students (88 female and 70 male), aged $18-30$ years $(M=21.82$, $S D=2.24)$, participated in the experiment. All participants had normal vision or corrected vision, and reported no stereo blindness.

\section{APPARATUS}

In order to maximize the ecological validity of research on mental rotation, this study focused on well- 


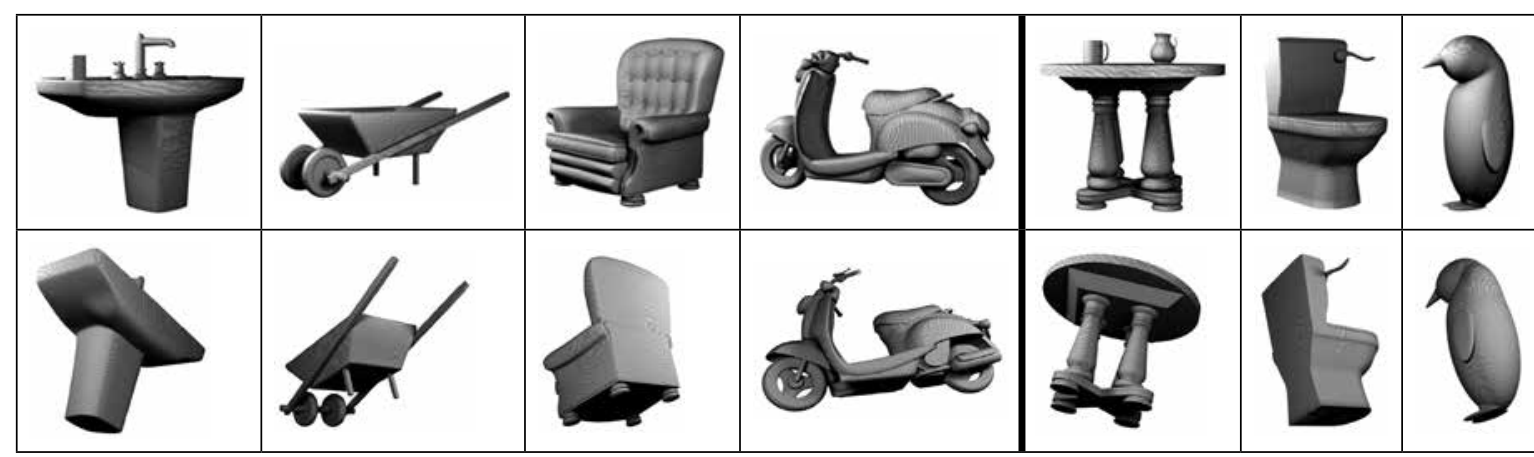

Figure 1. Prototypes of seven subordinate NMR categories presented in canonical orientation (upper row) and from an unusual viewpoint (lower row). The three objects on the right were excluded from the experiment (explanation in the text).

known three-dimensional objects presented not only in a plane (computer screen) but also stereoscopically as virtual objects, in their natural scenes. Three-dimensional objects were projected stereoscopically onto a $3.5 \times 2.30 \mathrm{~m}$ screen with the Inition ${ }^{\circledR}$ Duality Stereoscopic Projection System (for the first group) or in a plane on a 23" LCD monitor, 1280/1024 pixels (for the second group). The participants from the "stereo group" sat at a distance of approximately 3.35 $\mathrm{m}$ from the screen and watched the scene through polarizing glasses. The diameter of the circle into which all the rotated objects were entered was $34.6^{\circ}$ FOV. The participants from the "in-a-plane group" sat at a distance of $65 \mathrm{~cm}$ from the monitor and the diameter of the circle into which objects were entered was $24^{\circ} \mathrm{FOV}$. A program for presenting stimuli and collecting data was written using E-Prime. The participants responded via a three-key response keyboard which was comprised of the following labels: "the same" (if they decided that the rotated object was the same as the target); "different" (if they decided that the rotated object was different than the target); and "the next" (if they wanted to move on to the next part of the experiment). The experiment was carried out individually with each participant, in an acoustically isolated studio.

\section{STIMULI}

Four of the seven three-dimensional objects designed in natural colors via three-dimensional Studio $\mathrm{Max}^{\circledR}$ were used in the experiment. In the preliminary research, the participants performed a sequential version of the MRT on seven objects which, in the original position, were presented from the canonical or unusual point of view (see Figure 1) and on the plane or stereoscopically, analogous to the experimental trials.

All objects were presented in the same fully colored background of an exhibition hall in a contemporary art museum (see Figure 2). Target objects were rotated around the line of sight (not in depth). The axis of rotation was located at the center of mass of the object.

Based on the analysis of the differences in time and accuracy of the MRT execution for the seven objects, three of them were rejected from further study. They were characterized by a significantly longer or shorter RT-MRT and greater or lesser A-MRT than the other four objects. In order to verify the homogeneity of the set of selected stimuli, an analysis of variance (ANOVA) with the variable OBJECT [four different objects] as an intragroup factor was conducted. There were no significant differences between objects, both in terms of RT-MRT $[F(3,120)=0.73, M S E=0.06$, $p=.534,1-\beta=0.20]$ as well as A-MRT $[F(3,120)=2.39$, $M S E=0.03, p=.072,1-\beta=0.59]$. Only these four objects were used in the experiment.

\section{PROCEDURE}

The sequential version of the MRT was used in the experiment. After a one-second exposure of a gray mask, the target object was presented as long as the

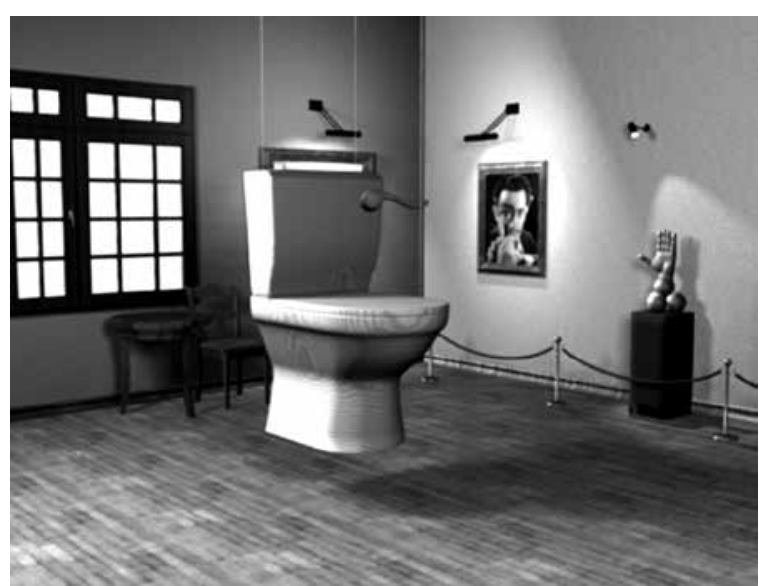

Figure 2. The exhibition hall of a contemporary art museum with the object-pattern in the center of the scene.
Rotation of well-known objects 
participant pressed a button. After the next $3 \mathrm{sec}-$ onds, the target object was presented in the position rotated or rotated and mirror-reversed. Each object was rotated ten times, at $60,120,180,240$, and 300 degrees. The presentation order of each pair of objects (target and rotated) was random. Participants then answered whether the object observed in the second position was only rotated or both rotated and mirror-reversed, in comparison to its original position. The answer (accuracy) and its latency (RT) were recorded.

\section{RESULTS}

The RT-MRT and the A-MRT were transformed using a logarithmic function to normalize the distribution of the data. Fitting the data to a normal distribution was confirmed through the Kolmogorov-Smirnov test (K-S test, $p>.05)$.

Reaction time of mental rotation task was tested using analysis of variance (ANOVA) with the variables ORIENTATION (Canonical vs. Unusual), PRESENTATION (In a Plane vs. Stereoscopic), and SEX (Female vs. Male) as the intergroup factors, and OBJECT (four objects), SAME-DIFFERENT (Rotation only vs. Rotation and Mirror-Reversed), and ANGLE OF ROTATION $\left(60^{\circ}, 120^{\circ}, 180^{\circ}, 240^{\circ}, 300^{\circ}\right)$ as intragroup factors.

There were no significant main effects of ORIENTATION $[F(1,150)=0.55, M S E=7.03, p=.459,1-\beta=0.11]$ or PRESENTATION $[F(1,150)=0.01, M S E=7.03$, $p=.922,1-\beta=0.05]$, but the findings showed a significant main effect of $\operatorname{SEX}[F(1,150)=7.60, M S E=7.03$, $\left.p=.007, \eta^{2}=.05\right]$ on the RT-MRT. Females needed significantly more time to execute the MRT than males. According to the within-subject factors, there were no significant main effects of the variables SAMEDIFFERENT $[F(1,150)=0.42, M S E=0.52, p=.518$,

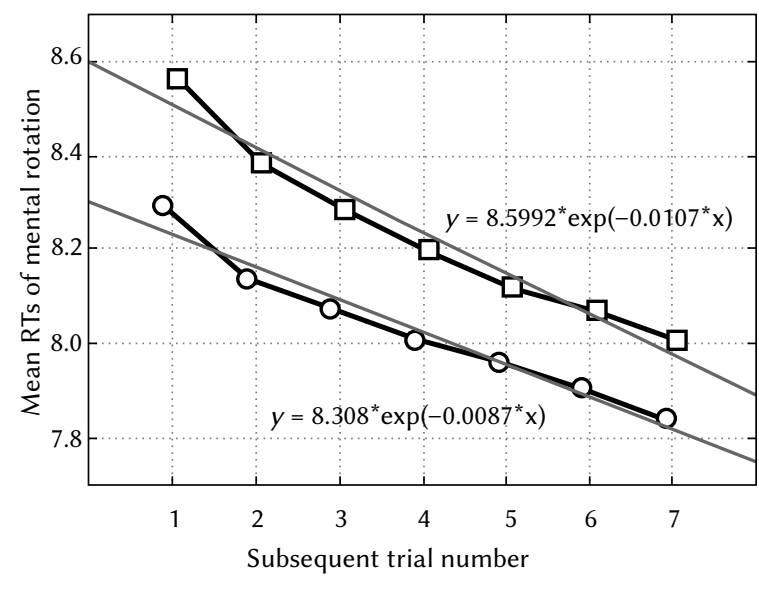

Orientation: -0 - Canonical $\neg \square-$ Unusual

Figure 3. The mean RTs of mental rotation of target objects presented in two orientations in seven consecutive trials. $1-\beta=0.10]$ or OBJECT $[F(3,450)=1.32, M S E=0.59$, $p=.267 ; 1-\beta=0.35]$ on the RT-MRT. However, the findings showed a significant main effect of the variable ANGLE OF ROTATION $[F(4,600)=36.39$, $\left.M S E=0.43, p<.001, \eta^{2}=.20\right]$ on the RT-MRT. This effect confirms the typical finding that the RT-MRT increases linearly with greater angular disparity between $0^{\circ}$ and $180^{\circ}$, and decreases between $180^{\circ}$ and $360^{\circ}$.

In order to analyze the effect of the variables ORIENTATION and PRESENTATION on the time of recognition of every object, post hoc Tukey HSD tests were conducted for the following averages: (1) time of watching each object in the original position; (2) the RT-MRT, separately for each object; and (3) the RT-MRT, separately for each object and for each angle of rotation. It was found that the only significant effect of the variables ORIENTATION and PRESENTATION was the time spent watching objects in the original position. The participants needed more time looking at each object presented in the original position from an unusual point of view $\left(\mathrm{M}_{\text {Unusual }}=2.497 \mathrm{~s}\right)$ than looking at this object presented in the original position from the canonical point of view $\left(\mathrm{M}_{\text {Canonical }}=1.999 \mathrm{~s}\right)$ (Tukey HSD test, $p<.001$ for each object). However, there were no sex differences in terms of the length of time watching objects from the canonical or unusual point of view, separately in each group.

Similarly, participants needed more time looking at the objects presented stereoscopically $\left(\mathrm{M}_{\text {Stereoscopic }}=3.359 \mathrm{~s}\right)$ than presented in a plane $\left(\mathrm{M}_{\text {Plane }}=1.486 \mathrm{~s}\right)$ (Tukey HSD test, $p<.001$ for each object). In line with the previous results, there were no sex differences in terms of the length of time watching objects presented stereoscopically or in a plane, separately in each group.

There were also no significant effects of the variables ORIENTATION and PRESENTATION on the RT-MRTs, separately for each object, or on the RTMRTs, separately for each object and for each angle of rotation (Tukey HSD test, $1.000>p>$.945).

Nevertheless, significant interaction effects of the variables ORIENTATION and ANGLE OF ROTA$\operatorname{TION}\left[F(4,600)=4.04, M S E=0.43, p=.003, \eta^{2}=.03\right]$, and PRESENTATION and ANGLE OF ROTATION $\left[F(4,600)=3.97, M S E=0.43, p=.003 ; \eta^{2}=.03\right]$ were found on the RT-MRT. The relationship between the RT-MRT and angular disparity was more pronounced with respect to the objects presented from the canonical point of view than from an unusual point of view, as well as when they were displayed stereoscopically than in a plane.

There were also some other significant interactions that shed new light on some of the main effects. The findings showed a significant interaction effect of the variables ORIENTATION and PRESENTATION on the RT-MRT $\left[F(1,150)=6.72, M S E=7.03, p=.011, \eta^{2}=.04\right.$; see Figure 3]. Post hoc analysis revealed a significant effect of the variable PRESENTATION on the RT-MRT 
for objects which, when in their original position, were viewed from an unusual point of view (Tukey HSD test, $p=.050$ ). The analysis also highlighted the significant effect of the variable ORIENTATION on the RT-MRT for objects presented stereoscopically (Tukey HSD test, $p=.038$ ). Moreover, a clear trend was found according to which the RT-MRT was shorter for objects which, in their original position, were viewed stereoscopically in canonical orientation than for objects presented in a plane (Tukey HSD test, $p=.085)$. However, there was no significant effect of the variable ORIENTATION on the RT-MRT for objects presented in a plane (Tukey HSD test, $p>.1$ ).

On the other hand, changing the orientation of the objects in the original position, from the canonical to the unusual, caused the execution of the MRT to be significantly longer in stereoscopic presentation than in a plane.

There was also a significant interaction effect of the variables SEX and ORIENTATION on the RT-MRT $\left[F(1,150)=4.23, M S E=7.03, p=.042, \eta^{2}=.03\right.$; see Figure 4]. Although females needed more time to complete the MRT than males, this effect concerned only the canonically oriented objects (Tukey HSD test; $p=.006)$. However, there were no differences between females and males in the RT-MRT for objects presented from an unusual point of view. Unlike women, men needed much more time to complete the MRT for objects presented in an unusual view, rather than the canonical orientation (Tukey HSD test, $p=.050$ ).

An analogous ANOVA with the variables ORIENTATION (Canonical vs. Unusual), PRESENTATION (In a Plane vs. Stereoscopic), and SEX (Female vs. Male) as the intergroup factors, and OBJECT (four objects), SAME-DIFFERENT (Rotation only vs. Rotation and Mirror-Reversed), and ANGLE OF ROTATION $\left(60^{\circ}, 120^{\circ}, 180^{\circ}, 240^{\circ}, 300^{\circ}\right)$ as intragroup factors was conducted for the A-MRT.

Contrary to the results for RT-MRT, significant main effects of the variables ORIENTATION $[F(1,150)=56.97$, $\left.M S E=0.61, p<.001, \eta^{2}=.28\right]$ and PRESENTATION $\left[F(1,150)=13.45, M S E=0.61, p<.001, \eta^{2}=.08\right]$ on the A-MRT were found. The MRT execution turned out to be much easier both when the objects were presented stereoscopically rather than in a plane, and when the target object in the original position was presented from the canonical rather than from an unusual point of view. However, there was no significant main effect of SEX $[F(1,150)=0.73, M S E=0.61, p=.395,1-\beta=0.14]$ on the A-MRT. Females and males performed the MRT equally accurately.

According to the within-subject factors, it appeared that all three variables had significant main effects on A-MRT: (1) SAME-DIFFERENT $[F(1,150)=11.39$, $\left.M S E=0.35, p<.001, \eta^{2}=.07\right] ;(2)$ ANGLE OF ROTATION $\left[F(4,600)=44.92, M S E=0.19, p<.001, \eta^{2}=.23\right]$; and (3) OBJECT $[F(3,450)=20.67, M S E=0.20, p<.001$, $\left.\eta^{2}=.12\right]$. The MRT execution turned out to be easier for objects which were only rotated than for objects which were rotated and mirror-reflected. The results also confirmed the well-known relationship between the angular disparity and A-MRT: the greater the angle of rotation, the higher the error rate. Despite the prior selection of objects for the experiment, differences between them were discovered, with respect to the A-MRT. In particular, it turned out that the MRT execution for one of the objects (wheelbarrow) was easier than for the other three. There were no significant differences between the other three objects due to the A-MRT execution. There were no sex differences in terms of error rate for objects presented stereoscopically or in a plane, as well as for objects oriented canonically or unusually in an original position, separately in each group.

Similarly as for the RT-MRT, a significant interaction effect of the variables ORIENTATION and ANGLE OF ROTATION on the A-MRT was found $\left[F(4,600)=2.74, M S E=0.19, p=.028, \eta^{2}=.02\right]$, but the interaction of variables PRESENTATION and ANGLE OF ROTATION was not significant $[F(4,600)=0.15$, $M S E=0.19, p=.964,1-\beta=.08]$. The relationship between the A-MRT and angular disparity was more pronounced with respect to the objects presented from the canonical point of view than from an unusual point of view. The U-shaped relationship between the A-MRT and angular disparity was the same for three-dimensional imaging and for presentation of objects in a plane.

\section{DISCUSSION}

The purpose of this study was to verify hypotheses concerning the effect of three-dimensional imaging and the canonicity of objects presented in the original position on the RT and the accuracy of the MRT

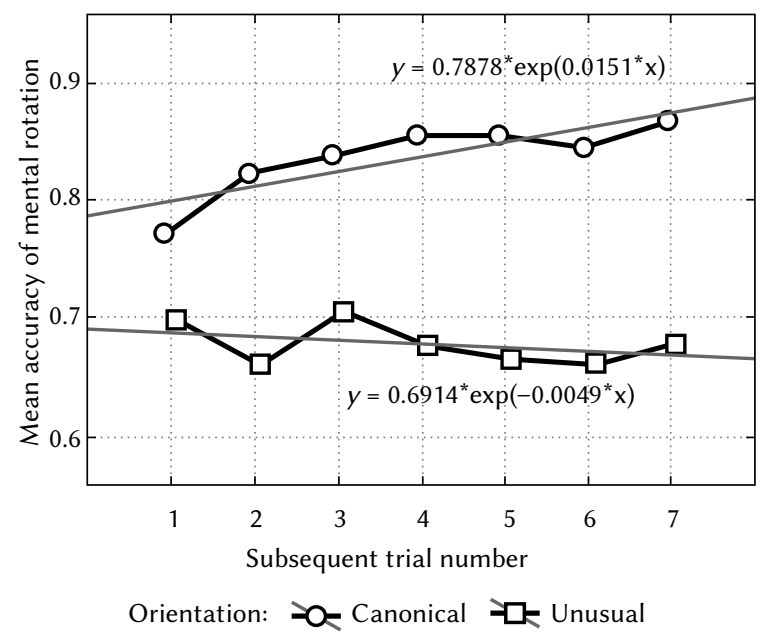

Figure 4. The mean accuracy of mental rotation of objects presented in successive trials in two orientations.
Rotation of well-known objects 
execution. The classical paradigm of the mental rotation task (MRT), developed by Shepard and Metzler (1971), was used in the experiment.

In order to verify the results of this study with the predictions of the mental rotation model, an analysis of the relationship between time and accuracy of MRT execution and angular disparity of rotated objects was conducted. As predicted by the mental rotation model, both the "U"-shaped A-MRT distribution and the inverted "U"-shaped RT-MRT distribution were found, due to the angular disparity (Shepard \& Cooper, 1982; Shepard \& Metzler, 1988). For the RT-MRT, this effect was more pronounced when the objects were displayed stereoscopically than in a plane, and when the objects were presented in the original position from the canonical orientation rather than an unusual point of view. On the other hand, in the case of the A-MRT, an effect of the ORIENTATION of objects presented in the original position on strengthening the relationship between accuracy and angular disparity was found. These effects have not previously been observed in studies of mental rotation which used three-dimensional objects, presented stereoscopically or in a plane. Mental rotation of stereoscopically presented objects with a smaller angle (e.g., $60^{\circ}$ or $300^{\circ}$ ) appears easier and faster than when they are presented in a plane. In turn, the MRT execution for stereoscopically presented objects rotated $180^{\circ}$ is slower and more difficult than for the objects presented in a plane.

Results of research on mental rotation usually show a significant sex difference (e.g., Jaušovec \& Jaušovec, 2012; Koscik, O’Leary, Moser, Andreasen \& Nopoulos, 2009; Kucian, Loenneker, Dietrich, Martin \& Von Aster, 2005; Parsons et al., 2004). In general, females need more time and make more mistakes during the MRT execution than males. The present study confirmed that females performed the MRT more slowly than males. However, there was also an interaction between the variables SEX and ORIENTATION. It was discovered that the significant difference between females and males concerns only those situations where a known object in the original position is seen from the canonical point of view (see Figure 4). The sex effect disappears when the object in the original position is seen from an unusual point of view. It is worth noting that in most research on mental rotation, objects in the original position are presented from the canonical point of view (e.g., Graf, 2006; Just et al., 2001, Zacks \& Michelon, 2005) and significant sex differences were evident. However, due to the fact that the shape of the object seen from an unusual point of view is atypical, the creation of its cognitive representation requires similar cognitive engagement, independent from sex.

Parson et al. (2004) found a significant effect of sex on RT-MRT, but only when the objects were "traditionally seen on paper-and-pencil measures", not in a virtual environment. The present study confirmed the traditional effect of sex on the RT-MRT execution, both when the objects were presented in a plane and stereoscopically. It is possible that the reason for differences between the results of the study by Parson et al. (2004) and the current research is the slightly different procedures for presentation of three-dimensional stimuli. Parson et al. (2004) used the technique of Virtual Reality (VR), which allows dynamic changes in vision of the area according to the movement of the head or body, while in our study, the static Duality Stereoscopic Projection System (DSPS) based on polarizing filters mounted in two projectors and the glasses was used. In the DSPS, the object is seen as three-dimensional, but only from one point of view.

There was no significant sex effect on the A-MRT, nor any significant interaction effects of the variable SEX with the variables ORIENTATION and PRESENTATION.

The differences between the RT-MRT for each object and for each angle of rotation are not significant. This means that neither the shape of the object, which is the basis for the recognition, nor its images after the rotation (regardless of whether this object was seen in the original position from a canonical or unusual perspective) affected the RT-MRT. In other words, the theory that differences in the RT-MRT for known objects are derived from the duration of their recognition can be rejected.

The present study mainly focused on the verification of the impact of the method of presentation of objects on the time and the accuracy of the MRT execution. Robert and Chevrier (2003) found that when objects are displayed stereoscopically, the RTMRT is shorter and A-MRT is higher in comparison to the objects displayed in a plane. In the present study we found that the main effect of the variable PRESENTATION on the RT-MRT was insignificant, while the effect of the variable PRESENTATION on the A-MRT was significant and consistent with the hypothesis. Analysis of the interaction of the variables PRESENTATION and ORIENTATION revealed the cause of the insignificant effect of the variable PRESENTATION on the RT-MRT. We confirmed the hypothesis that the shorter RT-MRT for stereoscopic presentation refers only to objects that are visible in the original position in a canonical orientation. If the MRT is performed on the objects seen in the original position in an unusual orientation, the stereoscopic presentation significantly prolongs its execution.

In Robert and Chevrier's (2003) study, among many other studies of mental rotation in which objects are presented stereoscopically or in a plane, Shepard-Metzler's cubes composing objects are used more often than well-known, real-world objects. In addition, Robert and Chevrier (2003) used a procedure of simultaneous presentation of objects in their original positions and after rotation, while the pres- 
ent study used a sequential variant of objects presented before and after rotation. Also, Robert and Chevrier (2003) did not control the canonicity orientation of objects in their original position.

We found a significant effect of the variable ORIENTATION on the A-MRT. The canonical exposure of objects in the original position significantly reduced the error rate during MRT execution, compared to the unusual presentation of objects in the original position. This result seems to confirm the hypothesis of Cutzu and Edelman (1994), according to which the MRT is essentially an operationalization of the categorization processes (rather than mental rotation or recognition) of an object when viewed from different viewpoints. The object in the original position acts as a category prototype. The similarities between results of the categorization tasks and the MRT also confirmed the results of neurophysiological studies (e.g. Farah, 1990; Shendan \& Stern 2007, 2008; Turnbull \& McCarthy, 1996; Warrington \& Taylor, 1973).

\section{CONCLUSIONS}

The results of this study indicate that three-dimensional imaging, as well as canonical or unusual orientation of a known object in the original position, has a significant impact on the accuracy and time of the MRT execution. Second, the inclusion of the method of presentation and orientation of objects in the experimental plan significantly modifies traditionally obtained results, according to which females have greater difficulty with the MRT execution than males. Thirdly, in this study, the homogeneity of the set of well-known and real-world objects used in the MRT was controlled because of the time and the accuracy of their recognition. However, the results obtained from this study indicate a significant influence of the variables ORIENTATION and PRESENTATION on RT-MRT and A-MRT, which, according to the classical theory of mental rotation, should not occur. This is probably a consequence of the fact that mental rotation theory has been developed almost exclusively based on data from studies that used drawings of cubes composing objects, rather than natural three-dimensional objects displayed stereoscopically. The results of this study indicate the need to conduct broader research and revise predictions arising from the theory of mental rotation in relation to well-known, natural objects. They also indicate the need for studies to verify the assumptions of mental rotation theory. The results of many studies support the hypothesis that the MRT may be an operationalization mental process of categorization, rather than mental rotation.

This study was supported by grant no. N106 064135 , to Piotr Francuz, from the Polish Ministry of Science and Higher Education.

\section{References}

Aitsiselmi, Y., \& Holliman, N. S. (2009). Using mental rotation to evaluate the benefits of stereoscopic displays. Stereoscopic Displays and Applications XX (2009) Proceedings of the SPIE Volume 7237.

Bauer, B., \& Jolicoeur, P. (1996). Stimulus dimensionality effects in mental rotation. Journal of Experimental Psychology: Human Perception and Performance, 22, 82-94.

Biederman, I., \& Gerhardstein, P. C. (1993). Recognizing depth-rotated objects: evidence and conditions for 3D viewpoint invariance. Journal of Experimental Psychology: Human Perception and Performance, 19, 1162-1182.

Bülthoff, H. H., Edelman, S. Y., \& Tarr, M. J. (1995). How are three-dimensional objects represented in the brain? Cerebral Cortex, 5, 247-260.

Corballis, M. C. (1988). Distinguishing clockwise from counterclockwise: does it require mental rotation? Memory \& Cognition, 16, 567-578.

Cutzu, F., \& Edelman, S. (1994). Canonical views in object representation and recognition. Vision $\mathrm{Re}$ search, 34, 3037-3056.

Cutzu, F., \& Edelman, S. (1998). Representation of object similarity in human vision: psychophysics and a computational model. Vision Research, 38, 2229-2257.

Edelman, S. (1995). Representation of similarity in three-dimensional object discrimination. Neural Computation, 7, 408-423.

Edelman, S., \& Bülthoff, H. H. (1992). Orientation dependence in the recognition of familiar and novel views of three-dimensional objects. Vision Research, 32, 2385-2400.

Farah, M. J. (1990). Visual agnosia: Disorders of object recognition and what they tell us about normal vision. Cambridge, MA: MIT Press.

Gaggioli, A., \& Breining, R. (2001). Perception and cognition in immersive virtual reality. In: G. Riva, F. Davide (eds.). Communications through virtual technologies: identity, community and technology in the communication age (pp. 71-86). Amsterdam: IOS Press.

Graf, M. (2006). Coordinate transformations in object recognition. Psychological Bulletin, 132, 920945.

Jaušovec, N., \& Jaušovec, K. (2012). Sex differences in mental rotation and cortical activation patterns: Can training change them? Intelligence, 40, 151162.

Jolicoeur, P. (1985). The time to name disoriented natural objects. Memory and Cognition, 13, 289303.

Jolicoeur, P., Regehr, S., Smith, L., \& Smith, G. (1985). Mental rotation of representations of two-dimensional and three-dimensional objects. Canadian Journal of Psychology, 39, 100-129.
Rotation of well-known objects 
Just, M. A., Carpenter, P. A., Maguire, M., Diwadkar, V., \& McMains, S. (2001). Mental rotation of objects retrieved from memory: a functional MRI study of spatial processing. Journal of Experimental Psychology: General, 130, 493-504.

Kanamori, N., \& Takeda, Y. (2003). The difference of mental processes between depth and plane rotation in natural objects. Technical Report on Attention and Cognition, 24, 1-4.

Konkle, T., \& Oliva, A. (2011). Canonical visual size for real-world objects. Journal of Experimental Psychology: Human Perception and Performance, 37, 23-37.

Koscik, T., O’Leary, D., Moser, D. J., Andreasen, N. C., \& Nopoulos, P. (2009). Sex differences in parietal lobe morphology: relationship to mental rotation performance. Brain Cognition, 69: 451-459.

Kucian, K., Loenneker, T., Dietrich, T., Martin, E., \& Von Aster, M. (2005). Gender differences in brain activation patterns during mental rotation and number related cognitive tasks. Psychology Science, 47, 112-131.

Lawson, R., \& Jolicoeur, P. (2003). Recognition thresholds for plane-rotated pictures of familiar objects. Acta Psychologica, 112, 17-41.

McWilliams, W., Hamilton, C. J., \& Muncer, S. J. (1997). On mental rotation in three dimensions. Perceptual and Motor Skills, 85, 297-298.

Murray, J. (1997). Flipping and spinning: spatial transformation procedures in the identification of rotated natural objects. Memory \& Cognition, 25, 96-105.

Neubauer, A. C., Bergner, S., \& Schatz, M. (2010). Twovs. three-dimensional presentation of mental rotation task: sex differences and effects of training on performance and brain activation. Intelligence, 38, 529-539.

Noudoost, B., Adibi, M., Moeeny, A., \& Esteky, H. (2005). Configural and analytical processing of familiar and unfamiliar objects. Cognitive Brain Research, 24, 436-441.

Palmer, S. E. (1999). Vision Science: Photons to Phenomenology. Cambridge, MA: MIT Press.

Palmer, S. E., Rosch, E., \& Chase, P. (1981). Canonical perspective and the perception of objects. In: J. Long, A. Baddeley (eds.). Attention and performance (vol. 9, pp. 135-151). Hillsdale, NJ: Erlbaum.

Parsons, L. M. (1995). Inability to reason an object's orientation using an axis and angle of rotation. Journal of Experimental Psychology: Human Perception and Performance, 21, 1259-1277.

Parsons, T. D, Larson, P., Kratz, K., Thiebaux, M., Bluestein, B., Buckwalter, J. G., \& Rizzo, A. A. (2004). Sex differences in mental rotation and spatial rotation in a virtual environment. Neuropsychologia, 42, 555-562.

Petit, L., Pegna, A., Mayer, E., \& Hauert, C. (2003). Representation of anatomical constraints in mo- tor imagery: Mental rotation of a body segment. Brain and Cognition, 51, 95-101.

Robert, M., \& Chevrier, E. (2003). Does men's advantage in mental rotation persist when real three-dimensional objects are either felt or seen? Memory and Cognition, 31, 1136-1145.

Ruddle, R., \& Jones, D. (2001). Manual and virtual rotation of a three-dimensional object. Journal of Experimental Psychology: Applied, 7, 286-296.

Schendan, H. E., \& Stern, C. E. (2008). Where vision meets memory: prefrontal-posterior networks for visual object constancy during categorization and recognition. Cerebral Cortex, 18, 1695-1711.

Schendan, H. E., \& Stern, C. E. (2007). Mental rotation and object categorization share a common network of prefrontal and dorsal and ventral regions of posterior cortex. Neuroimage, 35, 1264-1277.

Shepard, R. N., \& Cooper, L. A. (1982). Mental images and their transformations. Cambridge, MA: MIT Press.

Shepard, R. N., \& Metzler, J. (1971). Mental rotation of three-dimensional objects. Science, 171, 701-703.

Shepard, S., \& Metzler, D. (1988). Mental rotation: effects of dimensionality of objects and type of task. Journal of Experimental Psychology: Human Perception and Performance, 14, 3-11.

Tarr, M. J. (1995). Rotating objects to recognize them: a case study of the role of viewpoint dependency in the recognition of three-dimensional objects. Psychonomic Bulletin Review, 2, 55-82.

Tarr, M. J., \& Pinker, S. (1989). Mental rotation and orientation-dependence in shape recognition. Cognitive Psychology, 21, 233-282.

Turnbull, O. H., \& McCarthy, R. A. (1996). When is a view unusual? A single case study of orientation-dependent visual agnosia. Brain Research Bulletin, 40, 497-502.

Warrington, E. K., \& Taylor, A. M. (1973). The contribution of the right parietal lobe to object recognition. Cortex, 9, 152-164.

Zacks, J. M., \& Michelon, P. (2005). Transformations of visuospatial images. Behavioral and Cognitive Neuroscience Reviews, 4, 96-118.

Zacks, J. M., Mires, J., Tversky, B., \& Hazeltine, E. (2002). Mental spatial trans-formations of objects and perspective. Spatial Cognition and Computation, 2, 315-332. 